\title{
Derivation and validation of a nomogram model for pulmonary thromboembolism in patients undergoing lung cancer surgery
}

\author{
Yuping $\mathrm{Li}^{1} \wedge$, Lei Shen ${ }^{1}$, Junrong Ding ${ }^{1}$, Dong Xie ${ }^{1}$, Jian Yang ${ }^{1}$, Yanfeng Zhao ${ }^{1}$, Angelo Carretta ${ }^{2,3}$, \\ René Horsleben Petersen ${ }^{4}$, Sebastien Gilbert ${ }^{5}$, Yasuhiro Hida ${ }^{6}$, Servet Bölükbas ${ }^{7}$, Hiran C. Fernando ${ }^{8}$, \\ Gening Jiang ${ }^{1}$, Yuming Zhu' ${ }^{1}$
}

${ }^{1}$ Department of Thoracic Surgery, Shanghai Pulmonary Hospital, Tongji University School of Medicine, Shanghai, China; ${ }^{2}$ School of Medicine, Vita-Salute San Raffaele University, Milan, Italy; ${ }^{3}$ Department of Thoracic Surgery, IRCCS San Raffaele Scientific Institute, Via Olgettina 60, Milan, Italy; ${ }^{4}$ Department of Cardiothoracic Surgery, University Hospital of Copenhagen-Rigshospitalet, Copenhagen, Denmark; ${ }^{5}$ Division of Thoracic Surgery, Department of Surgery, The Ottawa Hospital, University of Ottawa, Ottawa, Canada; ${ }^{6}$ Department of Cardiovascular and Thoracic Surgery, Faculty of Medicine, Hokkaido University, Sapporo, Hokkaido, Japan; ${ }^{7}$ Department of Thoracic Surgery, Kliniken Essen-Mitte, Essen, Germany; ${ }^{8}$ Cardiothoracic Surgery, Allegheny General Hospital, Pittsburgh, PA, USA

Contributions: (I) Conception and design: Y Li, G Jiang, Y Zhu; (II) Administrative support: None; (III) Provision of study materials or patients: J Yang, G Jiang, Y Zhu; (IV) Collection and assembly of data: L Shen, J Ding, D Xie; (V) Data analysis and interpretation: Y Li, Y Zhao; (VI) Manuscript writing: All authors; (VII) Final approval of manuscript: All authors.

Correspondence to: Yuming Zhu; Gening Jiang. Department of Thoracic Surgery, Shanghai Pulmonary Hospital, Shanghai 200433, China. Email: zhuyuming@tongji.edu.cn; geningiiang@tongji.edu.cn.

\begin{abstract}
Background: A specific risk-stratification tool is needed to facilitate safe and cost-effective approaches to the prophylaxis of acute pulmonary thromboembolism (PTE) in lung cancer surgery patients. This study aimed to develop and validate a simple nomogram model for the prediction of PTE after lung cancer surgery using readily obtainable clinical characteristics.

Methods: A total of 14,427 consecutive adult patients who underwent lung cancer surgery between January 2015 and July 2018 in our institution were retrospectively reviewed. Included in the cohort were 136 patients who developed PTE and 544 non-PTE patients. The patients were randomly divided into the derivation group (70\%, 95 PTE patients and 380 non-PTE patients) and the validation group (30\%, 41 PTE patients and 164 non-PTE patients). A nomogram model was developed based on the results of multivariate logistic analysis in the derivation group. The cut-off values were defined using Youden's index. The prognostic accuracy was measured by area under the curve (AUC) values.

Results: In the derivation group, multivariate logistic analysis was carried out to evaluate the risk score. The risk assessment model contained five variables: age [95\% confidence interval (CI): 1.008-1.083, $\mathrm{P}=0.016$ ], body mass index (95\% CI: 1.077-1.319, $\mathrm{P}=0.001)$, operation time (95\% CI: $1.002-1.014, \mathrm{P}=0.008$ ), the serum level of cancer antigen 15-3 (CA15-3) before surgery (95\% CI: 1.019-1.111, $\mathrm{P}=0.005$ ), and the abnormal results of compression venous ultrasonography before surgery (95\% CI: 2.819-18.838, $\mathrm{P}<0.001$ ). All of them were independent risk factors of PTE. To simplify the risk assessment model, a nomogram model was established, which showed a good predictive performance in the derivation group (AUC 0.792, 95\% CI: 0.734-0.853) and in the validation group (AUC 0.813, 95\% CI: 0.737-0.890).

Conclusions: A high-performance nomogram was established on the risk factors for PTE in patients undergoing lung cancer surgery. The nomogram could be used to provide an individual risk assessment and guide prophylaxis decisions for patients. Further external validation of the model is needed in lung cancer surgery patients in other clinical centers.
\end{abstract}

Keywords: Pulmonary thromboembolism (PTE); lung cancer; risk assessment model; nomogram model

Submitted Oct 19, 2020. Accepted for publication Apr 21, 2021.

doi: $10.21037 /$ tlcr-21-109

View this article at: http://dx.doi.org/10.21037/tlcr-21-109

$\wedge$ ORCID: 0000-0001-5209-4131. 


\section{Introduction}

Venous thromboembolism (VTE) which includes deep vein thrombosis (DVT) and pulmonary thromboembolism (PTE) is a major global burden of disease. PTE is caused by a perfusion defect due to a thrombi dislodge from clots in vein walls and travel through the heart to pulmonary arteries (1). PTE is a common and potentially fatal disease in patients after surgery. The incidence of PTE in Asian populations is 15.9 per 100,000 person-years (2), which is much lower than that in Caucasian populations (71-117 per 100,000 person-years) (3-5). In China, the incidence of PTE is $0.1 \%$ (6). The overall 30 -day incidence of PTE after lung cancer surgery in our institution was $0.53 \%$ (7). Kentaroh reported 6 of 1,034 patients $(0.58 \%)$ with pulmonary malignant tumor developed PTE after surgery in their department (8). However, the incidence of PTE might be underestimated due to the lack of clinical symptoms and the high rates of misdiagnosis (9). Several studies have reported that patients with lung cancer are more likely to develop pulmonary embolism than patients with other solid tumors (10-13). Mason and Sugarbaker reported that venous thromboembolism is surprisingly common after pneumonectomy and portends poor survival $(14,15)$.

Studies have shown that patients who underwent thoracic surgery are usually at intermediate or high risk of PTE. Confirmed risk factors for PTE include patientrelated (usually permanent) risk factors such as age, obesity, smoking, hypercholesterolemia, hypertension, diabetes mellitus, active cancer, and a history of other diseases, and setting-related (usually temporary) risk factors such as surgical procedures, transfusion, central venous catheter, and chemotherapy (16-19).

Predicting the risk of recurrence in patients with VTE by developing simple nomograms (the Vienna Prediction Model and DAMOVES score) have been attempted in present studies (20-22). Parvizi et al. have reported that they used the national registry and institutional data to provide a simple nomogram for VTE prophylaxis after total joint arthroplasty (23). Shah et al. constructed nomograms to predict individual risk of 30-d VTE in high-risk general and thoracic surgery patients (24). But only $2.1 \%$ of the study population was after thoracic surgery. Compared with the present studies, we try to focus on postoperative acute PTE in patients undergoing lung cancer surgery. We believe that postoperative PTE prevention needs to take into account the differences in the risk profile of patients undergoing lung cancer surgery, and prophylaxis needs to be individualized.

Caprini has also developed a risk assessment model (RAM) that can identify patients at higher risk of VTE in the general surgical population (25). The Caprini risk assessment model, which is recommended by the guidelines of the American College of Chest Physicians (ACCP), is the most widely used model in surgical patients (17). However, the Caprini model has taken into account many risk factors and is a complicated tool for surgeons to use. In fact, in this model, all patients undergoing thoracic surgery are considered to be at a high or very high risks for development of PTE and are deemed to require chemical prophylaxis. Previous studies have developed models based on data derived from predominately Western populations $(13,17,26)$. Models for stratifying the risk of PTE in Asian populations are limited, especially for patients undergoing lung cancer surgery (2).

Therefore, we sought to develop an accurate, objective, and simple model for predicting PTE in patients who underwent lung cancer surgery using readily available clinical parameters, which would be helpful in guiding clinical decision-making on prophylaxis. We also internally validated the model in patients with acute symptomatic PTE. We present the following article in accordance with the transparent reporting of a multivariable prediction model for individual prognosis or diagnosis (TRIPOD) reporting checklist (available at http://dx.doi.org/10.21037/ tlcr-21-109) (27).

\section{Methods}

\section{Study design}

This study was a matched case-control study. The probability of exposure among sampled control patients was 0.2 and the correlation coefficient for exposure between matched case and control patients was 0.2. A sample of 136 PTE patients was obtained. For each PTE patient, a matched sample of 4 non-PTE patients was also obtained. This sample of 544 non-PTE patients achieved $90 \%$ power to detect an odds ratio of 2.0 versus the alternative of equal odds, using a Chi-squared test with the level of significance set at 0.05 . The study was conducted in accordance with the Declaration of Helsinki (as revised in 2013). This study was approved by the Institutional Review Board of Shanghai Pulmonary Hospital (No. K15-198) and was registered on ChiCTR (ChiCTR-RDC-15007490). All patients enrolled completed the informed consent form. 


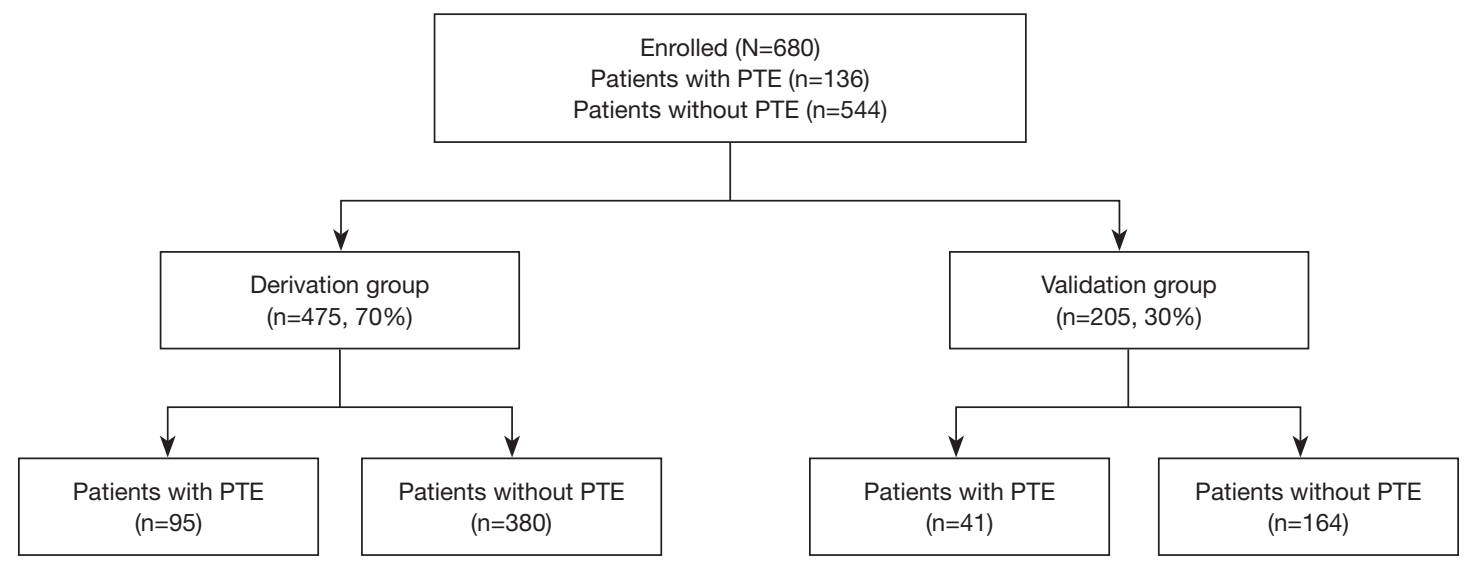

Figure 1 Group diagram of the study cohort. PTE, pulmonary thromboembolism.

\section{Patients and eligibility}

Data from a total of 14,427 lung cancer surgery patients were retrospectively analyzed. All consecutive adult patients who were diagnosed with PTE after lung cancer surgery between January 2015 and July 2018 in Shanghai Pulmonary Hospital were eligible for this study. The study was approved and a waiver for review of medical records was granted by Shanghai Pulmonary Hospital ethics committee. The incidence of PTE is less than 1\% (136/14,427). 136 consecutive cases who developed PTE within 1 month after lung cancer surgery and matched 4:1 with controls out of the total 14,291 control cases. 544 controls were randomly selected from all the lung cancer surgery patients without PTE within 1 month after lung cancer surgery. Enrolled patients were further randomized into derivation group (70\%) and validation group (30\%) (Figure 1).

The inclusion criteria for the PTE group were as follows: (I) 18 years or older; (II) inpatients who underwent lung cancer surgery via open or video-assisted thoracoscopic surgery; (III) the primary endpoint of the study was postoperative PTE, which was defined as occurrence of PTE within 30 days of lung cancer surgery. The primary diagnosis of PTE according to the International Classification of Diseases, $9^{\text {th }}$ Clinical Modification (ICD9-CM), or a secondary diagnosis of PTE with one of the following primary diagnoses that represent complications of PTE: respiratory failure, cardiogenic shock, cardiac arrest, syncope (16).

The exclusion criteria were as follows: (I) patients who had a history of VTE histories prior to surgery, (II) patients without complete clinical characteristics or follow-up data.

In this study, of the 136 PTE patients, 89 were diagnosed as PTE by computed tomography pulmonary angiography (CTPA), with single/bilateral/multi-lobar pulmonary artery embolism and its branches being the main type (Table S1). Seventeen patients who had personal history of DVT or PTE histories and five patients who were diagnosed with VTE by compression venous ultrasonography (CUS) or CTPA before surgery were excluded. A total of fifteen patients without complete clinical characteristics or followup data were also excluded.

\section{Clinical characteristics and follow-up}

Data were extracted from the patients' electronic medical records, which included demographic characteristics, medical history, surgical information, blood transfusion, length of stay, laboratory and imaging results. Our usual VTE prophylaxis consisted of low molecular weight heparin (4,000-6,000 international units once-daily). A total of 540/680 (79.4\%) of patients received this. CUS was undertaken in $97 / 136$ (71.3\%) of patients in the PTE group and $494 / 544(84.3 \%)$ of patients in the control group before operation. In our study, the abnormal results of lower limb vein by CUS includes venous blood stasis in the lower limbs or venous valve insufficiency. Perioperative blood transfusion is defined as blood transfusion during surgery and after surgery but does not include the transfusion after PTE occurrence. The abnormal results of ECG before surgery were defined as any kind of arrhythmias. Pulmonary dysfunction before surgery was defined as all kinds of abnormal lung function. In addition to lobectomy, extended pulmonary resection includes wedge resection, segmentectomy, lobectomies, tracheoplasty, angioplasty, 
partial chest wall resection, and pneumonectomy. The pathological results were reported by two professional pathologists. All patients were followed up by telephone, return visit, or inpatient observation, and the follow-up data were recorded until December 2018 or death.

\section{Derivation and validation of the PTE nomogram model}

The risk assessment model was developed by multivariate analyses. A receiver operating characteristics curve (ROC) and the area under the curve (AUC) was assessed to evaluate discriminatory ability. An initial analysis was performed based on patients with complete clinical data. For the main analysis, multiple imputation with chained equations was used to replace missing data for body mass index (BMI) values and $\mathrm{D}$-dimer test results before surgery. Variables with clinical significance and those with a $\mathrm{P}$ value of $<0.05$ in the univariate analyses were included in a backward stepwise multiple logistic regression analysis to identify the significant predictors of each outcome. Multivariate analyses were then carried out to evaluate independent risk factors associated with PTE. The Box-Tidwell method was used to test the linear correlation between the independent variables and logit conversion values. The Omnibus test of Model Coefficients and the Hosmer-Lemeshow goodnessof-fit test were used for integral evaluation, following which the logistic regression model was further simplified into a nomogram based on the coefficient values of related risk factors by the statistical program $\mathrm{R}$ version 3.4.2 (Ross Ihaka and Robert Gentleman, New Zealand). Further, to assess the discriminatory power of the nomogram model for predicting PTE, the area under the receiver operating characteristic curves (AUC) of the prediction model were compared in both groups and in the entire cohort. The possible value for an AUC ranges from 0.5 (no better discrimination than chance) to 1.0 (perfect discrimination).

\section{Statistical analyses}

Statistical analyses were performed with IBM-SPSS for Windows software, version 22 (SPSS Inc., Chicago, IL). Qualitative variables were expressed as numbers and percentages. Quantitative variables with normal distribution were expressed as the mean \pm standard deviation, and those with non-normal distribution were expressed as medians and quartiles. Comparisons between two groups were performed using the Chi-squared test for qualitative variables, Student's $t$-test for quantitative variables with normal distribution, or non-parametric test for quantitative variables with nonnormal distribution. The associations between independent risk factors and PTE were presented as odds ratios (OR) and their corresponding $95 \%$ confidence intervals (CI). A P value $<0.05$ was considered to be statistically significant. In the Hosmer-Lemeshow test, a $\mathrm{P}$ value $>0.05$ indicates that a model has high goodness of fit.

\section{Results}

\section{Characteristics and outcomes of PTE patients}

The incidence of PTE in the total patients after lung cancer surgery was less than $1 \%(136 / 14,427)$ in our institution. A total of 136 patients with PTE who underwent lung cancer surgery were stratified into intermediate-low risk $(\mathrm{n}=82)$, intermediate-high risk $(\mathrm{n}=34)$, and high-risk $(\mathrm{n}=20)$ subgroups according to the 2014 European Society of Cardiology (ESC) guidelines (Table 1) (16). There were no patients in the low-risk subgroup. The PTE patients had a mean age of 66 years. Of the 136 patients, 123 (91.9\%) cases presented with PTE alone, and PTE concomitant with DVT was diagnosed in $11(8.1 \%)$ cases. All of the patients experienced PTE within the first week after surgery. Dyspnea was the primary symptom in 123 (90.4\%) patients. In the high-risk subgroup, 13 patients presented with cardiac arrest as the main symptom. Increased plasma natriuretic peptide levels were reported in 45 (33.1\%) PTE patients, and $20(14.7 \%)$ cases had troponin I elevation. A total of 89 (65.4\%) patients were diagnosed with PTE by CTPA (Figure S1). After lung cancer surgery, 56 cases $(41.2 \%)$ were treated with 4,000 international units subcutaneous injection of low molecular weight heparin $(\mathrm{LMWH})$ once before diagnosis with PTE. After diagnosis with PTE, 130 cases $(95.6 \%)$ were treated with 6,000 international units subcutaneous injection of low molecular weight heparin (LMWH) once every 12 hours during hospitalization. Twenty-five patients received mechanical ventilation and 20 severe patients were treated with thrombolytic therapy (Table 1). Seventeen patients died of PTE, with a mortality rate of $12.5 \%$ among patients who developed PTE. Of note, in the high-risk subgroup, all 20 PTE patients received thrombolysis and their mortality rate was high (11/20, $55 \%)$.

\section{The risk factors associated with PTE}

To analyze the risk factors for PTE, the 136 patients with PTE and 544 patients without PTE were randomly divided into the 
Table 1 Risk stratification of patients with PTE undergoing lung cancer surgery

\begin{tabular}{|c|c|c|c|c|}
\hline Variables & Total $(n=136)$ & Intermediate-low ( $\mathrm{n}=82)$ & Intermediate-high $(\mathrm{n}=34)$ & High $(n=20)$ \\
\hline BMI, kg/m² & $25.11 \pm 2.76$ & $25.18 \pm 2.94$ & $24.62 \pm 2.27$ & $25.63 \pm 2.72$ \\
\hline Gender (males) & 67 & 38 & 20 & 9 \\
\hline Time of diagnose after surgery, days & $4 \pm 3$ & $4 \pm 4$ & $4 \pm 3$ & $2 \pm 1$ \\
\hline Dyspnea & 123 & 82 & 34 & 7 \\
\hline Cardiac arrest & 13 & 0 & 0 & 13 \\
\hline \multicolumn{5}{|l|}{ Clinical diagnosis, $n$} \\
\hline Confirmation by CTPA & 89 & 52 & 32 & 5 \\
\hline D-dimer before surgery (ng/mL) & $438 \pm 822$ & $484 \pm 947$ & $465 \pm 734$ & $209 \pm 118$ \\
\hline D-dimer after diagnose (ng/mL) & $5,013 \pm 6,735$ & $3,971 \pm 4,149$ & $4,430 \pm 5,883$ & $9,581 \pm 11,957$ \\
\hline Elevated BNP, $\mathrm{n}$ & 45 & 0 & 31 & 14 \\
\hline Elevated troponin I, n & 20 & 0 & 9 & 11 \\
\hline \multicolumn{5}{|l|}{ Treatments, $n$} \\
\hline Chemical prophylaxis & 56 & 33 & 16 & 7 \\
\hline Anticoagulant therapy & 130 & 82 & 34 & 14 \\
\hline Mechanical ventilation & 25 & 2 & 4 & 19 \\
\hline
\end{tabular}

BMI, body mass index; CTPA, computed tomography pulmonary angiography; BNP, B type natriuretic peptide.

derivation group (70\%, 95 PTE patients and 380 non-PTE patients) and the validation group (30\%, 41 PTE patients and 164 non-PTE patients) (Figure 1). Univariate and multivariate logistic analyses were performed, and 13 risk factors for PTE were subsequently identified (Table 2). Among them, age (OR: 1.045, 95\% CI: 1.008-1.083, P=0.016), BMI (OR: 1.192, 95\% CI: $1.077-1.319, \mathrm{P}=0.001$ ), operation time (OR: $1.008,95 \%$ CI: 1.002-1.014, $\mathrm{P}=0.008)$, cancer antigen 15-3 (CA15-3) level (OR: $1.064,95 \%$ CI: $1.019-1.111, \mathrm{P}=0.005$ ), and abnormal CUS results before surgery (OR: 7.287, 95\% CI: 2.819-18.838, $\mathrm{P}<0.001$ ) were found to be independently associated with PTE in patients after lung cancer surgery (Table 3). Furthermore, the risk score of PTE [Logit $(P)]$ could be calculated based on the coefficient values using the following formula: Logit $(P)$
$=-10.66+0.075 \times \mathrm{AGE}+0.206 \times \mathrm{BMI}+0.009 \times$ Operation time $+0.062 \times \mathrm{CA} 15-3+1.986 \times$ CUS

There was a significant linear correlation between the continuous variables and logit $(P)$ value $(\mathrm{P}=0.00556$, BoxTidwell). Further evaluation showed the model to have a high goodness of fit with significance $(\mathrm{P}<0.001$, Omnibus test; $\chi^{2}=10.515, \mathrm{P}=0.231>0.05$, Hosmer-Lemeshow goodness-of-fit test). This development regression model could correctly classify $85.8 \%$ of the cases.

\section{Development and validation of nomogram model}

Despite the logistic regression model having good prediction efficiency, it would be challenging for surgeons to 
Table 2 Multivariate analyses of risk factors for PTE in patients undergoing lung cancer surgery

\begin{tabular}{|c|c|c|c|c|}
\hline Risk factors & \multicolumn{2}{|c|}{ Results } & \multicolumn{2}{|c|}{ Adjusted results } \\
\hline Age & $1.045(1.005-1.086)$ & $0.025^{\star}$ & $1.045(1.008-1.083)$ & $0.016^{*}$ \\
\hline BMI & $1.195(1.072-1.333)$ & $0.001^{*}$ & $1.192(1.077-1.319)$ & $0.001^{*}$ \\
\hline Operation time & $1.005(0.998-1.013)$ & 0.134 & $1.008(1.002-1.014)$ & $0.008^{*}$ \\
\hline Abnormal results of CUS & $5.642(2.060-15.453)$ & $0.001^{*}$ & 7.287 (2.819-18.838) & $<0.001^{*}$ \\
\hline The level of D-dimer & $1.000(1.000-1.000)$ & 0.500 & - & - \\
\hline Blood loss during surgery & $1.001(0.999-1.004)$ & 0.298 & - & - \\
\hline Blood transfusion during surgery & $1.152(0.299-4.435)$ & 0.837 & - & - \\
\hline Thoracotomy & $0.837(0.281-2.493)$ & 0.749 & - & - \\
\hline Extended pulmonary resection & $0.937(0.409-2.148)$ & 0.878 & - & - \\
\hline TNM stage (later than IIb) & $1.364(0.662-2.811)$ & 0.400 & - & - \\
\hline
\end{tabular}

BMI, body mass index; CUS, compression venous ultrasonography; ECG, electrocardiogram. *, P $\leq 0.050$. The preoperative CUS specifically reference to preoperative CUS of lower limb vein. The abnormal results of lower limb vein by CUS includes venous blood stasis in the lower limbs or venous valve insufficiency. Perioperative blood transfusion is defined as blood transfusion during surgery and after surgery, but does not include the transfusion after PTE occurrence. The abnormal results of ECG before surgery were defined as any kind of arrhythmias. Pulmonary dysfunction before surgery was defined as all kinds of abnormal lung function. In addition to lobectomy, extended pulmonary resection includes wedge resection, segmentectomy, lobectomies, tracheoplasty, angioplasty, partial chest wall resection, and pneumonectomy.

Table 3 Independent risk factors for PTE in patients undergoing lung cancer surgery

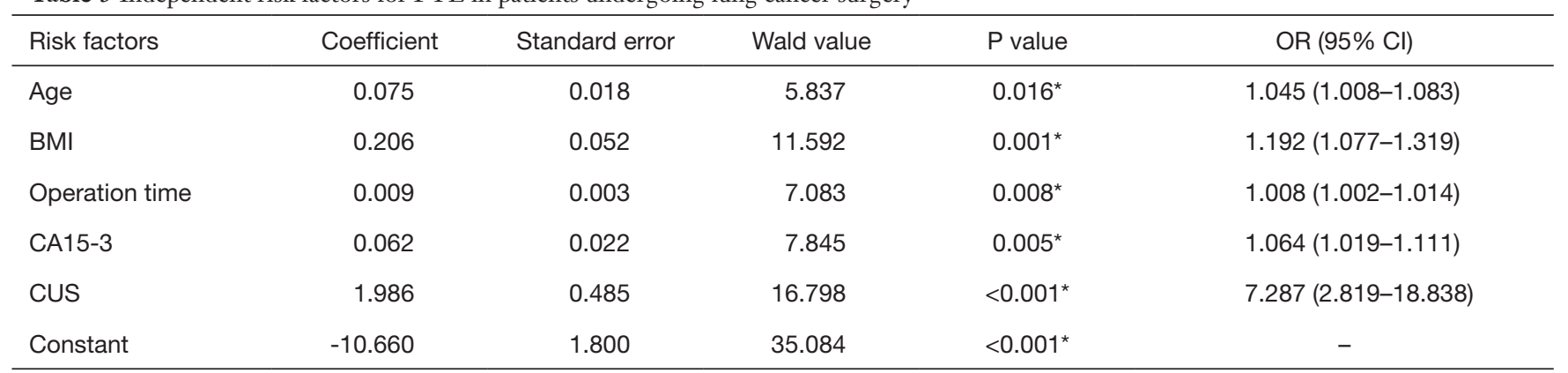

*, P $\leq 0.050$. BMI, body mass index; CUS, compression venous ultrasonography. CUS is a categorical variable, 0 indicates normal and 1 indicates abnormal results.

apply it in their daily clinical decision-making. To simplify the PTE risk assessment model, a nomogram model was established from the coefficient values of related risk factors. Example of how to apply the nomogram is given in the legend to Figure 2. First, the prediction efficiency of the nomogram model was assessed in the derivation group
( $\mathrm{n}=475$ ) by ROC curve analysis (Figure 3). The AUC was 0.792 (95\% CI: 0.734-0.853), and the cut-off for total score was 131 points. Based on this cut-off value, the sensitivity, specificity, and other evaluation indicators of the model were determined in the validation group $(n=205)$ and in the entire cohort $(\mathrm{n}=680)$. The results are shown in Figure 3 
Points

AGE

BMI

Operation time

CA153

CUS

Total Points

Linear Predictor

Risk

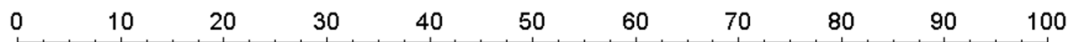

$\begin{array}{llllllllllllllllll}25 & 30 & 35 & 40 & 45 & 50 & 55 & 60 & 65 & 70 & 75 & 80 & 85 & 90 & & & & \\ 12 & 14 & 16 & 18 & 20 & 22 & 24 & 26 & 28 & 30 & 32 & 34 & 36\end{array}$

\begin{tabular}{llllllllll}
\hline 0 & 50 & 100 & 150 & 200 & 250 & 300 & 350 \\
\hline 0 & 5 & 10 & 15 & 20 & 25 & 30 & 35 & 40 & 45 \\
\hline
\end{tabular}

0

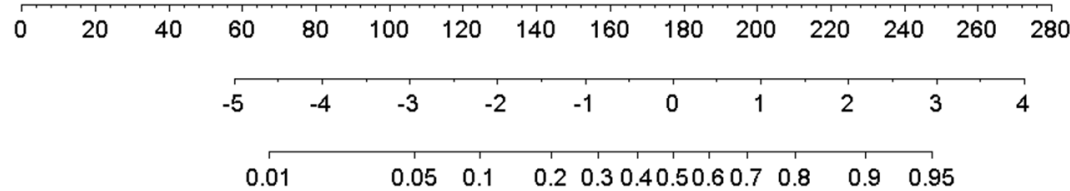

Figure 2 A nomogram model for PTE risk in patients undergoing lung cancer surgery. AGE (years); BMI, body mass index (kg/m²); Operation time (min); CA15-3, the serum level of carbohydrate antigen CA15-3 before surgery (U/mL); CUS, the results of compression venous ultrasonography before surgery. CUS is a categorical variable, 0 indicates normal results and 1 indicates abnormal results. Instructions: Locate the patient's age on the Age axis. Draw a line upward to the Points axis to determine the points. Repeat the same process for the other predictor variables. Sum all the points from the variables and locate it on the Total Points axis. Draw a line down to the Risk of PTE axis to determine the patient's probability of developing PTE. For instance, a 75 -year-old patient with a BMI of $30 \mathrm{~kg} / \mathrm{m}^{2}$ had an operation time of $200 \mathrm{minutes}$, a CA15-3 level of $11 \mathrm{U} / \mathrm{mL}$, and abnormal result of CUS before surgery (value $=1$ ). The score of each variable was 52, 74, 36, 15, and 46 points, respectively; thus, the patient had a total score of 223 points, and the risk of developing PTE was 0.88 .

A

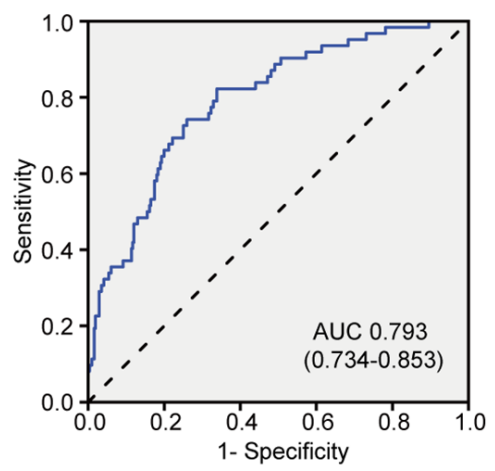

B



C

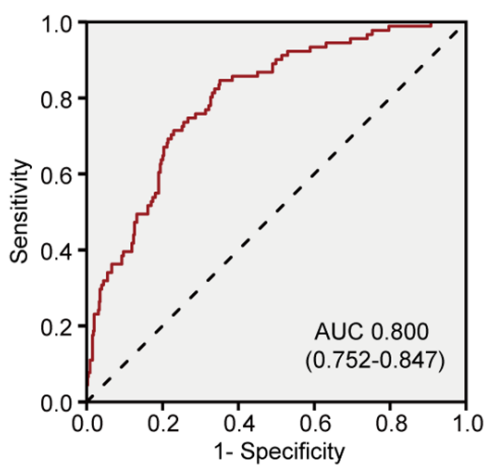

Figure 3 Charts display the Youden Threshold (specificity, sensitivity). Panel (A) shows the ROC curve according to the nomogram for predicting PTE of patients who underwent lung cancer in the derivation group. Panel (B) shows the ROC curve according to the nomogram for predicting PTE of patients who underwent lung cancer in the validation group. Panel (C) shows the ROC curve according to the nomogram for predicting PTE of patients who underwent lung cancer in the entire cohort. AUC, the area under the curve; ROC, receiver operating characteristic. 
Table 4 Validation of the nomogram model for PTE in patients undergoing lung cancer surgery

\begin{tabular}{lccc}
\hline Indicators & Derivation group (N=475) & Validation group (N=205) & Total group (N=680) \\
\hline AUC $(95 \% \mathrm{Cl})$ & $0.793(0.734-0.853)$ & $0.813(0.737-0.890)$ & $0.800(0.752-0.847)$ \\
Sensitivity & $82.3 \%$ & $89.7 \%$ & $84.6 \%$ \\
Specificity & $66.1 \%$ & $62.0 \%$ & $64.9 \%$ \\
Mistake diagnostic rate & $33.9 \%$ & $38.0 \%$ & $35.1 \%$ \\
Omission diagnostic rate & $17.7 \%$ & $10.3 \%$ & $15.4 \%$ \\
Total consistent rate & $68.8 \%$ & $66.9 \%$ & $68.2 \%$ \\
Youden index & $48.4 \%$ & $51.7 \%$ & $49.5 \%$ \\
Odd product & 9.1 & 14.2 & 10.2 \\
Positive predictive value & $32.3 \%$ & $33.3 \%$ & $32.6 \%$ \\
Negative predictive value & $95.0 \%$ & $96.6 \%$ & $95.5 \%$ \\
\hline AUC, the area & & \\
\hline
\end{tabular}

AUC, the area under the curve.

and Table 4. The AUCs in the validation group and entire cohort were 0.813 (95\% CI: $0.737-0.890)$ and 0.800 (95\% CI: $0.752-0.847)$, respectively, which indicated the good prediction efficiency of the model.

\section{Discussion}

PTE is a very severe complication after thoracic surgery. Studies have reported the prevalence of VTE after lobectomy to range from $0.2-20 \%(26,28-30)$. As the most severe clinical manifestation of VTE, PTE is the main cause of death after thoracic surgery. On average, the 136 lung cancer patients enrolled in this study were diagnosed with PTE within 4 days after surgery. The patients with a high risk of PTE were diagnosed PTE, on average, within 2 days after surgery. Systemic thrombolysis as initial therapy is currently recommended by the 2016 American College of Chest Physicians and 2014 European Society of Cardiology guidelines only for patients with acute massive or high-risk PTE; i.e., those presenting with hemodynamic compromise, broadly defined as a systolic blood pressure of less than $90 \mathrm{mmHg}$ (16,31). In patients with acute PTE were initial treated with lowmolecular-weight heparin ( $\mathrm{LMWH}$ ), we gave twice-daily administration for at least 5 days and 3 months long-term treatment of PTE with oral anticoagulants or vitamin $\mathrm{K}$ antagonists (VKAs) treatment. In this study, systemic thrombolysis was administered in 20/136 (14.7\%) of patients in high risk PTE group and anticoagulant therapy were treated in 132/136 (95.6\%) of patients.
The mechanism of PTE is complex and includes patientrelated risk factors, as well as transient surgical risk factors. In this study, we systematically evaluated all the potential risk factors of PTE in Chinese patients who received lung cancer surgery, and finally identified five risk factors as being independently associated with PTE.

According to the results of the logistic regression analyses, the risk of PTE increases by $4.5 \%$ for every year increase in age. In line with the results of previous studies involving non-Chinese populations, older patients were found to have an increased risk of VTE (32,33). Another study in an Asian population with non-small cell lung cancer also reported that age was an independent risk factor for VTE (34). BMI is another patient-related, usually permanent, PTE risk factor. Numerous studies have revealed that obese patients carry a higher risk of deep vein thrombosis and pulmonary embolism (18,19,35-40), while being underweight is associated with a reduced risk of VTE (36). In this study, the OR value of obesity was 1.192 (95\% CI: 1.077-1.319). BMIs over 35 are extremely rare in Chinese populations; thus, the BMI stratification in the modified Caprini model is not suitable for Chinese patients (25). In fact, one of the reasons we developed this new nomogram model was to show the linear correlation between BMI and PTE risk in a Chinese population.

Aside from patient-related risk factors, three settingrelated, usually temporary, PTE risk factors were also identified: operation time, CA15-3 level and abnormal CUS results before surgery. Abnormal results of CUS include venous stasis or venous valve insufficiency. In 
general, lung cancer surgery increases the postoperative risk of PTE due to the long operation time and associated trauma, as well as the large number of days patients can stay in bed. In our study, the surgery-related risk factors of PTE, such as thoracotomy, pulmonary resection, operation time, central venous catheter, and tumor, node, metastasis (TNM) stage of lung cancer, were included in univariate and multivariate analyses. However, only operation time was identified as one of the independent PTE risk factors. Similar results were also reported by Kim and Cui $(41,42)$. In our study, of all the serum tumor biomarker, CA15-3 has shown an association with the risk of PTE (95\% CI: 1.019-1.111, $\mathrm{P}=0.005)$, though the mechanism is unknown. The preoperative serum levels of CA15-3, an important biomarker of breast cancer, is also elevated in patients with lung cancer $(43,44)$. Although the levels of CA15-3 in patients with and without PTE were in the normal range, patients with PTE were observed to have significantly higher levels of CA15-3 than patients without PTE, and further logistic regression analysis revealed that the risk of PTE increased by $6.4 \%$ for every $1 \mathrm{U} / \mathrm{mL}$ increase in the level of CA15-3 before surgery. Similarly, Yu et al. showed that carbohydrate antigens, CA 15-3 may be useful for differential diagnosis and prediction of malignancies in patients with IDVT (45). Further investigation should focus on confirming the value of CA 15-3 as risk factor in patients with PTE. Abnormal CUS results before surgery were also an independent PTE risk factor in our study. The proportion of PTE patients with abnormal CUS results before surgery was significantly higher than that of patients without PTE (13.7\% vs. $2.6 \%, \mathrm{P}<0.001)$. In addition, some studies have indicated a similar correlation between increased baseline D-dimer level and the occurrence of VTE $(46,47)$. In our study, the baseline D-dimer level in patients with PTE was significantly higher than that in patients without PTE $(463 \pm 846$ vs. $254 \pm 813 \mu \mathrm{g} / \mathrm{L}, \mathrm{P}<0.001)$. However, logistic regression analysis revealed that baseline D-dimer level was not an independent risk factor for PTE after lung cancer surgery; consequently, it was not included in the model.

The selection of variables is the most important part of developing a model. During the selection process, all recognized risk factors should be taken into consideration, along with other potential variables. Too many variables will increase the difficulty in developing the model and limit the model's clinical application. In our study, five clinical risk factors were easily obtained from the patients' electronic medical records, and the nomogram model had a good prediction efficiency. The PTE risk for individuals could be conveniently and effectively predicted, which indicates that our model could be used to guide clinical decision-making on prophylaxis for PTE and reduce bleeding complications caused by unnecessary use of anticoagulants. Our study demonstrated that the patients with scores over 131 points should ideally receive anticoagulant thromboprophylaxis with low-molecular-weight heparin (LMWH) or low-dose unfractionated heparin (LDUH) during hospitalization and extended PTE prophylaxis after discharge. For patients after lung cancer surgery with bleeding or with a high risk for major bleeding, we suggest mechanical thromboprophylaxis with graduated compression stockings (GCS) or intermittent pneumatic compression (IPC) (17).

However, this study was limited by its non-randomized, retrospective design. The data used to develop the nomogram were obtained from a single clinical center; therefore, the model needs to be further validated in patients from other clinical centers. Until our model has been externally validated in other populations, we recommend that it is only used for patients undergoing lung cancer surgery.

\section{Conclusions}

In this study, age, BMI, operation time, the serum level of CA15-3 before surgery, and abnormal results of CUS before surgery were shown to be independent risk factors for PTE in patients who underwent lung cancer surgery. A nomogram model was established based on these five risk factors and could predict the risk of PTE effectively. More data from other clinical centers are needed to further validate the clinical applicability of this nomogram model.

\section{Acknowledgments}

The authors appreciate the academic support from AME Lung Cancer Collaborative Group.

Funding: None.

\section{Footnote}

Reporting Checklist: The authors have completed the TRIPOD reporting checklist. Available at http://dx.doi. org/10.21037/tlcr-21-109

Data Sharing Statement: Available at http://dx.doi. org/10.21037/tlcr-21-109

Conflicts of Interest: All authors have completed the ICMJE 
uniform disclosure form (available at http://dx.doi. org/10.21037/tlcr-21-109). RHP receive speaker fee from Medtronic and Advisory Board AstraZeneca. The authors have no other conflicts of interest to declare.

Ethics Statement: The authors are accountable for all aspects of the work in ensuring that questions related to the accuracy or integrity of any part of the work are appropriately investigated and resolved. The study was conducted in accordance with the Declaration of Helsinki (as revised in 2013). The study was approved by institutional ethics board of Shanghai Pulmonary Hospital Research Ethics Board (No. K15-198) and registered on ChiCTR (ChiCTR-RDC-15007490). All patients enrolled completed the informed consent form.

Open Access Statement: This is an Open Access article distributed in accordance with the Creative Commons Attribution-NonCommercial-NoDerivs 4.0 International License (CC BY-NC-ND 4.0), which permits the noncommercial replication and distribution of the article with the strict proviso that no changes or edits are made and the original work is properly cited (including links to both the formal publication through the relevant DOI and the license). See: https://creativecommons.org/licenses/by-nc-nd/4.0/.

\section{References}

1. Di Nisio M, van Es N, Buller HR. Deep vein thrombosis and pulmonary embolism. Lancet 2016;388:3060-73.

2. Lee CH, Lin LJ, Cheng CL, et al. Incidence and cumulative recurrence rates of venous thromboembolism in the Taiwanese population. J Thromb Haemost 2010;8:1515-23.

3. Spencer FA, Emery C, Lessard D, et al. The Worcester Venous Thromboembolism study: a populationbased study of the clinical epidemiology of venous thromboembolism. J Gen Intern Med 2006;21:722-7.

4. Silverstein MD, Heit JA, Mohr DN, et al. Trends in the incidence of deep vein thrombosis and pulmonary embolism: a 25-year population-based study. Arch Intern Med 1998;158:585-93.

5. Anderson FA Jr, Wheeler HB, Goldberg RJ, et al. A population-based perspective of the hospital incidence and case-fatality rates of deep vein thrombosis and pulmonary embolism. The Worcester DVT Study. Arch Intern Med 1991;151:933-8.

6. Yang Y, Liang L, Zhai Z, et al. Pulmonary embolism incidence and fatality trends in chinese hospitals from 1997 to 2008: a multicenter registration study. PLoS One 2011;6:e26861.

7. Li YP, Shen L, Huang W, et al. Prevalence and Risk Factors of Acute Pulmonary Embolism in Patients with Lung Cancer Surgery. Semin Thromb Hemost 2018;44:334-40.

8. Miyoshi K, Okumura N, Kokado Y, et al. Pulmonary thromboembolism in patients after surgery for pulmonary malignant tumor. Kyobu Geka 2006;59:821-5.

9. Yang Y, Wang N, Zhai Z, et al. The analysis of diagnosis and treatment of inpatients with pulmonary thromboembolism in Beijing. Zhonghua Jie He He Hu Xi Za Zhi 2014;37:898-902.

10. Ma SQ, Lin Y, Ying HY, et al. Solid malignancies complicated with pulmonary embolism: clinical analysis of 120 patients. Chin Med J (Engl) 2010;123:29-33.

11. Chew HK, Davies AM, Wun T, et al. The incidence of venous thromboembolism among patients with primary lung cancer. J Thromb Haemost 2008;6:601-8.

12. Connolly GC, Menapace L, Safadjou S, et al. Prevalence and clinical significance of incidental and clinically suspected venous thromboembolism in lung cancer patients. Clin Lung Cancer 2013;14:713-8.

13. Walker AJ, Card TR, West J, et al. Incidence of venous thromboembolism in patients with cancer - a cohort study using linked United Kingdom databases. Eur J Cancer 2013;49:1404-13.

14. Mason DP, Quader MA, Blackstone EH, et al.

Thromboembolism after pneumonectomy for malignancy: an independent marker of poor outcome. J Thorac Cardiovasc Surg 2006;131:711-8.

15. Sugarbaker DJ, Jaklitsch MT, Bueno R, et al. Prevention, early detection, and management of complications after 328 consecutive extrapleural pneumonectomies. J Thorac Cardiovasc Surg 2004;128:138-46.

16. Konstantinides SV, Torbicki A, Agnelli G, et al. 2014 ESC guidelines on the diagnosis and management of acute pulmonary embolism. Eur Heart J 2014;35:3033-69, 69a-69k.

17. Kearon C, Akl EA, Comerota AJ, et al. Antithrombotic therapy for VTE disease: Antithrombotic Therapy and Prevention of Thrombosis, 9th ed: American College of Chest Physicians Evidence-Based Clinical Practice Guidelines. Chest 2012;141:e419S-96S.

18. Kucher N, Tapson VF, Goldhaber SZ, et al. Risk factors associated with symptomatic pulmonary embolism in a large cohort of deep vein thrombosis patients. Thromb 
Haemost 2005;93:494-8.

19. Pomp ER, le Cessie S, Rosendaal FR, et al. Risk of venous thrombosis: obesity and its joint effect with oral contraceptive use and prothrombotic mutations. Br J Haematol 2007;139:289-96.

20. Eichinger S, Heinze G, Jandeck LM, et al. Risk assessment of recurrence in patients with unprovoked deep vein thrombosis or pulmonary embolism: the Vienna prediction model. Circulation 2010;121:1630-6.

21. Marín-Romero S, Elías-Hernández T, Asensio-Cruz MI, et al. Risk Of Recurrence After Withdrawal Of Anticoagulation In Patients With Unprovoked Venous Thromboembolism: External Validation Of The Vienna Nomogram And The Dash Prediction Score. Arch Bronconeumol 2019;55:619-26.

22. Franco Moreno AI, Garcia Navarro MJ, Ortiz Sanchez J, et al. A risk score for prediction of recurrence in patients with unprovoked venous thromboembolism (DAMOVES). Eur J Intern Med 2016;29:59-64.

23. Parvizi J, Huang R, Rezapoor M, et al. Individualized Risk Model for Venous Thromboembolism After Total Joint Arthroplasty. J Arthroplasty 2016;31:180-6.

24. Shah DR, Wang H, Bold RJ, et al. Nomograms to predict risk of in-hospital and post-discharge venous thromboembolism after abdominal and thoracic surgery: an American College of Surgeons National Surgical Quality Improvement Program analysis. J Surg Res 2013;183:462-71.

25. Caprini JA. Risk assessment as a guide to thrombosis prophylaxis. Curr Opin Pulm Med 2010;16:448-52.

26. Christensen TD, Vad H, Pedersen S, et al. Venous thromboembolism in patients undergoing operations for lung cancer: a systematic review. Ann Thorac Surg 2014;97:394-400.

27. Collins GS, Reitsma JB, Altman DG, et al. Transparent reporting of a multivariable prediction model for individual prognosis or diagnosis (TRIPOD): the TRIPOD statement. BMJ 2015;350:g7594.

28. Gómez-Hernández MT, Rodríguez-Pérez M, NovoaValentín N, et al. Prevalence of venous thromboembolism in elective thoracic surgery. Arch Bronconeumol 2013;49:297-302.

29. Kalweit G, Huwer H, Volkmer I, et al. Pulmonary embolism: a frequent cause of acute fatality after lung resection. Eur J Cardiothorac Surg 1996;10:242-6; discussion 246-7.

30. White RH, Zhou H, Romano PS. Incidence of symptomatic venous thromboembolism after different elective or urgent surgical procedures. Thromb Haemost 2003;90:446-55.

31. Kearon C, Akl EA, Ornelas J, et al. Antithrombotic Therapy for VTE Disease: CHEST Guideline and Expert Panel Report. Chest 2016;149:315-52.

32. Folsom AR, Lutsey PL, Nambi V, et al. Troponin T, NTproBNP, and venous thromboembolism: the Longitudinal Investigation of Thromboembolism Etiology (LITE). Vasc Med 2014;19:33-41.

33. Tsai AW, Cushman M, Rosamond WD, et al. Cardiovascular risk factors and venous thromboembolism incidence: the longitudinal investigation of thromboembolism etiology. Arch Intern Med 2002;162:1182-9.

34. Lee YG, Kim I, Lee E, et al. Risk factors and prognostic impact of venous thromboembolism in Asian patients with non-small cell lung cancer. Thromb Haemost 2014;111:1112-20.

35. Ageno W, Becattini C, Brighton T, et al. Cardiovascular risk factors and venous thromboembolism: a meta-analysis. Circulation 2008;117:93-102.

36. Delluc A, Mottier D, Le Gal G, et al. Underweight is associated with a reduced risk of venous thromboembolism. Results from the EDITH case-control study. J Thromb Haemost 2009;7:728-9.

37. Holst AG, Jensen G, Prescott E. Risk factors for venous thromboembolism: results from the Copenhagen City Heart Study. Circulation 2010;121:1896-903.

38. Samama MM. An epidemiologic study of risk factors for deep vein thrombosis in medical outpatients: the Sirius study. Arch Intern Med 2000;160:3415-20.

39. Severinsen MT, Kristensen SR, Johnsen SP, et al. Anthropometry, body fat, and venous thromboembolism: a Danish follow-up study. Circulation 2009;120:1850-7.

40. Wattanakit K, Lutsey PL, Bell EJ, et al. Association between cardiovascular disease risk factors and occurrence of venous thromboembolism. A time-dependent analysis. Thromb Haemost 2012;108:508-15.

41. Cui S, Li H, Tian B, et al. Risk Factors Associated with Venous Thromboembolism after Lung Cancer Surgery: A Single-center Study. Zhongguo Fei Ai Za Zhi 2018;21:753-60.

42. Kim JY, Khavanin N, Rambachan A, et al. Surgical duration and risk of venous thromboembolism. JAMA Surg 2015;150:110-7.

43. Li X, Xu Y, Zhang L. Serum CA153 as biomarker for cancer and noncancer diseases. Prog Mol Biol Transl Sci 2019;162:265-76. 
44. Wu H, Wang Q, Liu Q, et al. The Serum Tumor Markers in Combination for Clinical Diagnosis of Lung Cancer. Clin Lab 2020;66. doi: 10.7754/Clin.Lab.2019.190533.

45. Yu M, Wang YH, Abdalla AME, et al. Carbohydrate antigens as potential biomarkers for the malignancy in patients with idiopathic deep venous thrombosis: a retrospective cohort study. J Huazhong Univ Sci
Technolog Med Sci 2014;34:722-8.

46. Sendama W, Musgrave KM. Decision-Making with D-Dimer in the Diagnosis of Pulmonary Embolism. Am J Med 2018;131:1438-43.

47. Tritschler T, Kraaijpoel N, Le Gal G, et al. Venous Thromboembolism: Advances in Diagnosis and Treatment. JAMA 2018;320:1583-94.
Cite this article as: Li Y, Shen L, Ding J, Xie D, Yang J, Zhao Y, Carretta A, Petersen RH, Gilbert S, Hida Y, Bölükbas S, Fernando HC, Jiang G, Zhu Y. Derivation and validation of a nomogram model for pulmonary thromboembolism in patients undergoing lung cancer surgery. Transl Lung Cancer Res 2021;10(4):1829-1840. doi: 10.21037/tlcr-21-109 
Supplementary

Table S1 Univariate analyses of risk factors for PTE in patients undergoing lung cancer surgery

\begin{tabular}{|c|c|}
\hline Variablec & Derivatior \\
\hline valiadies & Patients with PTE (n=95) \\
\hline Age, years & $65 \pm 7$ \\
\hline $\mathrm{BM}, \mathrm{kg} / \mathrm{m}^{2}$ & $25.41 \pm 2.80$ \\
\hline Operation time, min & $163 \pm 61$ \\
\hline Blood loss during surgery, $\mathrm{mL}$ & $173 \pm 228$ \\
\hline length of stay, days & $21 \pm 10$ \\
\hline D-dimer before surgery, $\mu \mathrm{g} / \mathrm{L}$ & $463 \pm 846$ \\
\hline Blood routine & \\
\hline Hemoglobin, g/L & $133 \pm 14$ \\
\hline Red blood cells, $\times 10^{12} \mathrm{~g} / \mathrm{L}$ & $4.45 \pm 0.44$ \\
\hline Leukocyte, $\times 10^{9} \mathrm{~g} / \mathrm{L}$ & $6.34+1.68$ \\
\hline Neutrophil, \% & $59.0 \pm 9.6$ \\
\hline Platelet, $\times 10^{\circ} \mathrm{g} / \mathrm{L}$ & $219 \pm 51$ \\
\hline Hematocrit, L/L & $40.7 \pm 3.8$ \\
\hline Biochemical routine & \\
\hline$\gamma$-glutamyl aminotransferase, $\mathrm{U} / \mathrm{L}$ & $34 \pm 30$ \\
\hline Glutamic-pyruvic transaminase, IU/L & $21 \pm 12$ \\
\hline Aspartate aminotransferase, IU/L & $21 \pm 8$ \\
\hline Total Protein, g/L & $68 \pm 5$ \\
\hline Albumin, $g / L$ & $40 \pm 4$ \\
\hline Globulin, g/L & $28 \pm 3$ \\
\hline Prealbumin, $\mathrm{mg} / \mathrm{L}$ & $250 \pm 54$ \\
\hline Uric acid, $\mu$ mol/L & $314 \pm 77$ \\
\hline Urea nitrogen, mmo/L & $5.6 \pm 1.6$ \\
\hline Creatinine, umol/L & $67 \pm 17$ \\
\hline Glucose, mmol/L & $5.9 \pm 1.9$ \\
\hline Potassium, mmol/L & $4.0 \pm 0.4$ \\
\hline Sodium, mmo/L & $142 \pm 3$ \\
\hline Chlorine, mmol/L & $103 \pm 3$ \\
\hline Calcium, mmol/L & $2.30 \pm 0.13$ \\
\hline Tumor biomarkers & \\
\hline Alpha fetoprotein, $\mu \mathrm{g} / \mathrm{mL}$ & $5.87 \pm 2.84$ \\
\hline Carcinoembryonic antigen, ug/L & $7.59 \pm 16.46$ \\
\hline Neuron-specific enolase, ng/L & $13.94 \pm 4.73$ \\
\hline Gastrin-releasing peptide precursor, pg/mL & $19.35 \pm 4.44$ \\
\hline B2-microglobulin, $\mu \mathrm{g} / \mathrm{mL}$ & $1.25 \pm 0.45$ \\
\hline Ferritin, ng/mL & $77.59 \pm 56.04$ \\
\hline Carbohydrate antigen CA242, U/mL & $8.29 \pm 5.27$ \\
\hline Carbohydrate antigen CA153, U/mL & $14.39 \pm 9.39$ \\
\hline CYFRA21-1, ng/mL & $3.25 \pm 6.67$ \\
\hline CA50, U/mL & $8.76 \pm 8.13$ \\
\hline Carbohydrate antigen CA199, U/mL & $11.91 \pm 22.36$ \\
\hline Carbohydrate antigen CA72-4, U/mL & $1.57 \pm 0.48$ \\
\hline Squamous cell carcinoma antigen, ng/mL & $1.12 \pm 0.22$ \\
\hline Categorical variables & \\
\hline Gender & \\
\hline Male & $49(51.6 \%)$ \\
\hline Female & $46(48.4 \%)$ \\
\hline Compression venous ultrasonography & \\
\hline Abnormal results & $13(19.7 \%)$ \\
\hline Venous valve insufficiency & $13(13.7 \%)$ \\
\hline Venous stasis & $0(0 \%)$ \\
\hline DVT & $0(0 \%)$ \\
\hline Not checked & $29(30.5 \%)$ \\
\hline Treatment history & \\
\hline Hormone therapy & $2(2.1 \%)$ \\
\hline Chemotherapy & $2(2.1 \%)$ \\
\hline History of disease & \\
\hline Other tumors & $5(5.3 \%)$ \\
\hline Fracture & $1(1.1 \%)$ \\
\hline PTE or DVT & $1(1.1 \%)$ \\
\hline Varicose veins & $4(4.2 \%)$ \\
\hline Hypertension & $18(86 \%)$ \\
\hline Diabetes & $9(9.5 \%)$ \\
\hline Heart disease & $1(1.1 \%)$ \\
\hline Cerebral infarction & $1(1.1 \%)$ \\
\hline Abnormal electrocardiogram & $18(22.0 \%)$ \\
\hline Pulmonary dysfunction & $27(28.4 \%)$ \\
\hline Chemoprophylaxis & $35(36.8 \%)$ \\
\hline Mortality & $14(14.7 \%)$ \\
\hline Surgical data & \\
\hline Type of procedure & \\
\hline Thoracotomy & $20(21.2 \%)$ \\
\hline Video-assisted thoracoscopic surgery & 75 (78.9\%) \\
\hline Sublobectomy & $15(15.8 \%)$ \\
\hline Lobectomy & $50(52.6 \%)$ \\
\hline Extended pulmonary resection & $30(31.6 \%)$ \\
\hline Blood type & \\
\hline A & $33(34.7 \%)$ \\
\hline B & $26(27.4 \%)$ \\
\hline 0 & $24(25.3 \%)$ \\
\hline AB & $12(12.6 \%)$ \\
\hline Pathology & \\
\hline Adenocarcinoma & $70(73.7 \%)$ \\
\hline Non-adenocarcinoma & $25(26.3 \%)$ \\
\hline Multiple primary lung cancer & $11(11.6 \%)$ \\
\hline TNM stage (UICC and AJCC, version 7) & $2(2.1 \%)$ \\
\hline 0 & \\
\hline la & $45(47.4 \%)$ \\
\hline lb & $26(27.4 \%)$ \\
\hline Ila & $5(5.3 \%)$ \\
\hline III & $1(1.1 \%)$ \\
\hline IIIIa & $15(15.8 \%)$ \\
\hline IV & $1(1.1 \%)$ \\
\hline Blood transfusion & $17(17.9 \%)$ \\
\hline Central venous catheter & $83(87.4 \%)$ \\
\hline
\end{tabular}





Figure S1 Representative CTPA images in patients with PTE. Panel (A) highlights an embolism in the left pulmonary artery on CTPA (white arrow, axial view). Panel (B) highlights multi embolisms in the right pulmonary artery and lobar arteries on CTPA (white arrow, axial view). Panel (C) highlights bilateral embolisms in both main pulmonary artery and an embolism in main pulmonary artery (white arrows, axial view). Panel (D) shows axial view of the main pulmonary artery. A long thrombus ridded across the pulmonary trunk. 